\title{
Inter-specific and Developmental Differences on the Array of Antennal Chemoreceptors in Four Species of Triatominae (Hemiptera: Reduviidae)
}

\author{
M Gracco, S Catalá ${ }^{+}$
}

\author{
CRILAR, Mendoza y Entre Ríos, Anillaco, 5301 La Rioja, Argentina
}

The aim of the work was to investigate the pattern of chemoreceptor sensilla in adults and fifth stage nymphs of Rhodnius prolixus, R. neglectus, Triatoma infestans and $\mathrm{T}$. sordida in order to study differences and similarities between genera and species. Three types of sensilla were analyzed by light microscopy: thin-walled trichoidea, thick-walled trichoidea and basiconica. The number of sensilla of each three types were counted. The length of the antennal segments were also used as a variable for the analysis. The statistical analysis showed that the number of these antennal chemoreceptors had significant differences between species and between adults and nymphs of each species. Discriminant analysis separates incompletely the fifth stage nymphs of the four species and showed similarity between them. Discriminant analysis performed with 12 variables of the antennae, allowed a complete separation of the adults of the four species.

Key words: sensilla - chemoreceptors - Triatominae - Rhodnius prolixus - Rhodnius neglectus - Triatoma infestans - Triatoma sordida

Triatominae are widely distributed in the Americas. All of them are obligate bloodfeeders and many are important as vectors of Trypanosoma cruzithe causative agent of Chagas disease or American trypanosomiasis (Schofield 1994). Their mechanism of host finding and orientation is not understood in detail, but involves detection of $\mathrm{CO}_{2}$, warmth (probably as radiant heat) and a series of odour stimuli including ammonia, and lactic, butyric and pyruvic acids (Bernard 1974, Lazzari 1990, Lorenzo Figueiras et al. 1994, Taneja \& Guerin 1997). The antenna is the main organ devoted to the detection of such stimuli.

The Triatominae are equipped with a pair of four-segmented antennae bearing a variety of sensilla including several types of chemoreceptors and mechanoreceptors, as well as putative thermohygroreceptors (Wigglesworth an\& Gillet 1934, Bernard 1974, Catalá 1994, 1997, Catalá \& Schofield 1994, Catalá et al. 1998). A recent study of the antennal sensilla of five triatomine genera using scanning electron microscopy (Catalá 1997) dem-

\footnotetext{
The project received financial support from CONICOR, British Council, RED ECLAT and SECYT Universidad Nacional de Córdoba.

${ }^{+}$Corresponding author. Fax: +54 03827-494231. E-mail: scatala@crilar.com.ar

Received 15 June 1999

Accepted 5 August 1999
}

onstrated inter-specific differences in sensilla density and pattern of distribution. Developmental differences had been observed in Triatoma sordida and Rhodnius pictipes, but only sexual differences in $T$. sordida. Developmental and sexual changes are related to basiconica and trichoidea thin- and thick-walled sensilla, which are considered as chemoreceptors.

Complete morphometric data of the antennae and its sensilla are of paramount importance to the understanding of the specificity thresholds and transduction processes of an antennal sensory system (Zacharuk 1985). Former works about triatomine sensilla pattern (Catalá \& Schofield 1994, Catalá 1996, 1997) used density of sensilla and were based on numerous photographs of scanning electron microscopy, a rather expensive approach. In the present paper, the use of light microscopy allowed the study of the sensilla pattern from the whole antennae and the estimation of the total population of chemoreceptors.

The multiporous thin-walled trichoidea sensilla were first described in T. infestans by Bernard (1974). Electrophysiological studies showed that they respond to direct breathing (Mayer 1968) a range of fatty acids, particularly pyruvic and lactic acids, and to amyl acetate (Bernard 1974). They are superficially similar to the E1, E2 and D sensilla of the bedbug, Cimex lectularius (Steinbrecht \& Muller 1976). The basiconica sensilla, which are also multiporous but double walled, have recently been implicated in the reception of $\mathrm{NH}_{3}$ (Taneja \& Guerin 
1997) and fatty acid molecules. They also respond to changes in temperature and humidity (Type F, Bernard 1974) and are similar to the $C$ receptors in C. lectularius responding to fatty acids (Steinbrecht $\&$ Muller 1976). In the case of the single-pored thick-walled trichoidea sensilla however, we neither have a clear indication of function nor the stimuli to which they respond in Triatominae. Morphologically, they are similar to the trichoidea sensilla described for other insects (Steinbrecht \& Muller 1976, Schaller 1978). From the general description of these sensilla given by Zacharuck (1985) we can suppose them to be gustatory or contact chemoreceptors, primarily detecting molecules in liquid phase.

The aim of this work was to investigate the pattern of chemoreceptor sensilla in adults and fifth stage nymphs of $R$. prolixus, $R$. neglectus, $T$. infestans and T. sordida in order to study differences and similarities between genera and species. The four species of Triatominae selected for the study have primitive silvatic habitats, but all of them can invade domestic and peridomestic habitats. $R$. prolixus is considered the primary domestic vector of T. cruzi in Venezuela, Colombia and parts of Central America, while R. neglectus occurs mainly in the cerrado region of central Brazil and invades houses less frequently (Barret 1991). T. sordida is known from a wide range of silvatic habitats in Brazil, Paraguay, Bolivia and Argentina, where it frequently occupies peridomestic habitats and occasionally forms domestic colonies. T. infestans is believed to originate from silvatic populations amongst rockpiles in central Bolivia, but it has been transported as a domestic species over a wide area of southern Latin America where it is the primary vector of T. cruzi.

\section{MATERIALS AND METHODS}

The insects - We examined the antennae of 80 specimens: 10 males and 10 fifth stage nymphs of each four species of Triatominae: $R$. prolixus, $R$. neglectus, T. infestans and T. sordida. All had been reared at approximately $28^{\circ} \mathrm{C}$ and $60 \%$ RH in laboratory colonies at the Servicio Nacional de Chagas, Punilla (Cordoba), where they were fed fortnightly on chickens.

Microscopical preparations - Antennae from nymphs and adult bugs were cut from the head at the level of the antennal tubercle, and prepared for gross morphological studies. Antennae were diaphanised in 3\% $\mathrm{NaOH}$ (Lazzari 1990) mounted in glycerine and examined under oil immersion using an ocular micrometer for measurements.

Sensilla analysis - Recognition of the different sensilla by light microscopy was made with reference to the classification of Catalá and Schofield (1994), Catalá (1997) and unpublished
SEM photomicrographs from those works provided by S Catalá (cf. Fig. 1). The three main types of chemoreceptor (basiconica, thin and thick walled trichoidea) were counted from the whole antennal preparations, excluding the scape, as neither adults nor nymphs hold chemoreceptors on this proximal segment. The length of each of the three distal antennal segments was also measured.

Differences between means were compared by unifactorial ANOVA and Tuckey's test (Sokal \& Rohlf 1979). Discriminant analysis allowed studying the multivariate relationships between species and Mahalanobis distances were used for measure the distance between two points in the space defined by two or more correlated variables.

\section{RESULTS}

Table I shows segment length and total chemoreceptors from the four species. The second and more distal flagellar segment was the most populated area in Rhodnius species but not in Triatoma where the first flagellar segment holds more than one thousand of chemoreceptors, in both species. The chemoreceptors number on pedicel varied from zero in Rhodnius to several hundreds in Triatoma. The total number of antennal chemoreceptors showed no correlation with the length of the antenna $(n=40, r=0.36, p>0.05)$.

Fig. 1 shows details of the three types of chemoreceptors studied here. The total number of sensilla trichoidea and basiconica showed significant differences between species and between adults and nymphs, with nymphs always showing fewer receptors than the adults (Table II). Rhodnius adults had considerably fewer receptors compared to adults of Triatoma. Nymphs of T. sordida showed fewer sensilla than nymphs of the other species, whereas adult $T$. sordida showed many more than adults of the other species.

The thick-walled trichoidea were the most abundant sensilla in adults and nymphs. The two species of Triatoma showed similar number of thickwalled sensilla, but $T$. sordida had a significantly higher population of thin-walled sensilla $(\mathrm{P}<0.001)$. The two species of Rhodnius had significantly fewer trichoidea sensilla compared to Triatoma. Data from the 40 adult specimens (four species) showed a strong correlation between total thick walled and total thin walled sensilla $(\mathrm{r}=0.82, \mathrm{t}=8.95$, $\mathrm{p}<0.001)$. No additional correlationships had been detected.

The fifth stage nymphs showed chemoreceptors only on the distal end of the second segment of the flagellum. Using four variables from this segment (number of basiconica, trichoidea thin and thick walled and length of flagellum 2) a discrimi- 

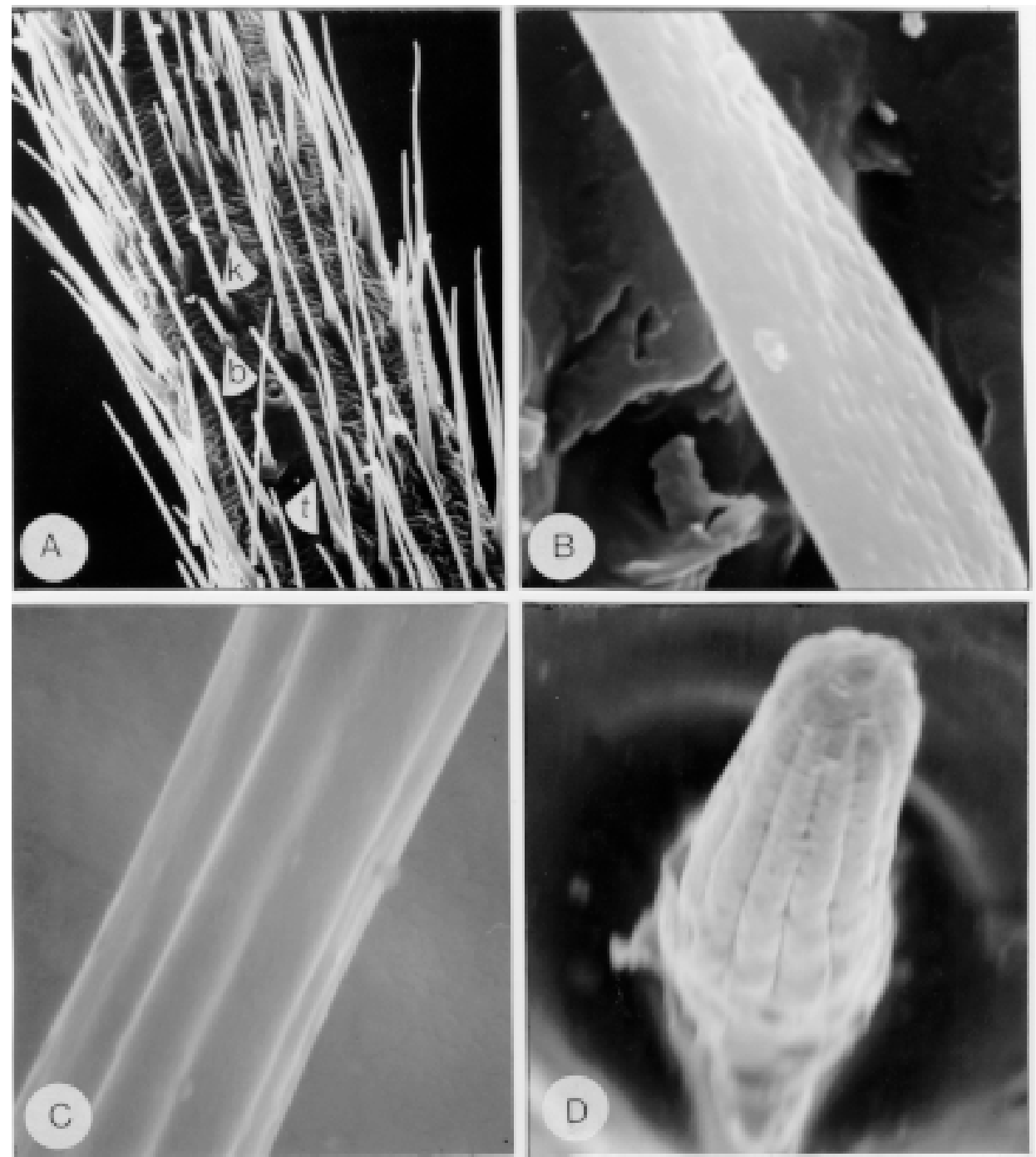

Fig. 1: (A) Triatoma sordida. Scanning electron micrograph of the tip of second flagellar segment showing the different types of chemo-sensilla. b: basiconic; t: thin walled, k: thick walled x 500; (B) Triatoma infestans. Thin walled trichoidea, detail of the wall showing pores. $\mathrm{x} 8000$; (C) Rhodnius prolixus: thick walled trichoidea, detail of the wall. $\mathrm{x}$ 20000; (D) Rhodnius neglectus. Sensillum basiconica: grooved wall and terminal pore (x 13000).

\section{TABLE I}

Lenght (L) (mm) of the segment and total number $(\mathrm{N})$ of chemoreceptors on the three distal segments of Rhodnius prolixus, R. neglectus, Triatoma infestans and T. sordida antenna. Mean (standard deviation)

\begin{tabular}{lcccccccc}
\hline & \multicolumn{2}{c}{ Triatoma sordida } & \multicolumn{2}{c}{ T. infestans } & \multicolumn{2}{c}{ Rhodnius neglectus } & \multicolumn{2}{c}{ R. prolixus } \\
\cline { 2 - 9 } Segment & $\mathrm{L}$ & $\mathrm{N}$ & $\mathrm{L}$ & $\mathrm{N}$ & $\mathrm{L}$ & $\mathrm{N}$ & $\mathrm{L}$ & $\mathrm{N}$ \\
\hline Pedicel & 2.66 & $890^{\mathrm{C}}$ & 3.72 & $222^{\mathrm{D}}$ & 3.25 & $0^{*}$ & 2.88 & $0^{*}$ \\
adult & $(0.12)$ & $(217)$ & $(0.19)$ & $(40)$ & $(0.11)$ & - & $(0.11)$ & - \\
Flagel I & 2.02 & $1159^{\mathrm{A}}$ & 3.47 & $1286^{\mathrm{A}}$ & 1.26 & $572^{\mathrm{B}}$ & 1.56 & $720^{\mathrm{B}}$ \\
adult & $(0.1)$ & $(168)$ & $(0.22)$ & $(166)$ & $(0.05)$ & $(92)$ & $(0.07)$ & $(161)$ \\
Flagel II & 1.56 & $765^{\mathrm{E}}$ & 2.51 & $938^{\mathrm{F}}$ & 1.64 & $903^{\mathrm{F}}$ & 1.65 & $954^{\mathrm{F}}$ \\
adult & $(0.11)$ & $(62)$ & $(0.17)$ & $(142)$ & $(0.06)$ & $(78)$ & $(0.10)$ & $(211)$ \\
Flagel II & 0.87 & 453 & 1.38 & 609 & 1.05 & 524 & 0.97 & 639 \\
nymph & $(0.05)$ & $(43)$ & $(0.09)$ & $(93)$ & $(0.03)$ & $(40)$ & $(0.05)$ & $(112)$ \\
\hline
\end{tabular}

A differs with $\mathrm{B} ; \mathrm{C}$ and $\mathrm{D}$ differ with * and between them; $\mathrm{E}$ differ with $\mathrm{F}$. All at $\mathrm{p}<0.001$. 
TABLE II

Number of three types of chemoreceptor sensilla on the antennae of Rhodnius prolixus, R. neglectus, Triatoma infestans and T. sordida

\begin{tabular}{lcrrrrrrr} 
& \multicolumn{2}{c}{ Triatoma sordida } & \multicolumn{2}{c}{ T. infestans } & \multicolumn{2}{c}{ Rhodnius neglectus } & \multicolumn{2}{c}{ R. prolixus } \\
\cline { 2 - 8 } & Adult & Nymph & Adult & Nymph & Adult & Nymph & Adult & Nymph \\
\hline Thin & 756 & 44 & 524 & 48 & 275 & 69 & 226 & 44 \\
walled & $(75)$ & $(10.8)$ & $(95.3)$ & $(11.7)$ & $(43)$ & $(10.7)$ & $(67)$ & $(22)$ \\
Thick & 1851 & 358 & 1762 & 497 & 1074 & 406 & 1273 & 539 \\
walled & $(303)$ & $(37)$ & $(180)$ & $(81)$ & $(91.4)$ & $(31.6)$ & $(267)$ & $(84.4)$ \\
Basiconica & 220 & 51 & 146 & 64 & 124 & 49 & 185 & 56 \\
& $(53.7)$ & $(4.7)$ & $(45)$ & $(16.6)$ & $(39.1)$ & $(5.3)$ & $(51)$ & $(16)$ \\
\hline Total & 2827 & 453 & 2432 & 609 & 1473 & 524 & 1684 & 639 \\
\hline
\end{tabular}

nant analysis did not separate completely the fifth stage nymphs of the four species. When the analysis was performed excluding the variable: "length of flagellum 2", the Mahalanobis distances showed that nymphs of $T$. sordida are similar to $R$. neglectus (Mahalanobis distance: 4.74) and, T. infestans stay very close to $R$. prolixus (Mahalanobis distance: 1.74) (Fig. 2). Grouping T. infestans and $R$. prolixus and $T$. sordida and $R$. neglectus, the number of thick walled trichoidea differed significantly between groups (mean $[\mathrm{T} . \mathrm{i}+\mathrm{R} . \mathrm{p}]=518, \mathrm{SD}=83.5$; mean [T.s+R.n] $=382, \mathrm{SD}=41 ; \mathrm{p}<0.00001)$. Data from the 40 fifth nymphal stage specimens (four species) showed no correlation between total thick walled and total thin walled sensilla.

Comparison of chemoreceptor number between nymphs and adults indicates a much higher transitional increment for the thin walled trichoidea in the two species of Triatoma where this population rises 11 times in $T$. infestans and 17 times in $T$. sordida. The transitional increment is not significantly different between genera or species for basiconica sensilla; it differs significantly between

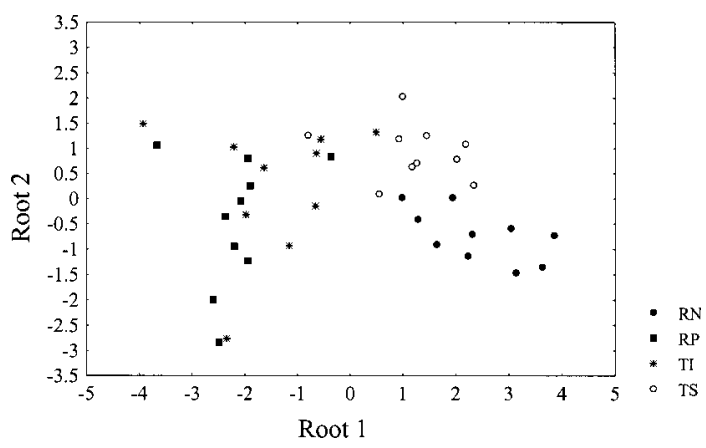

Fig. 2: discriminant analysis performed with four variables of the nymphs antennae. Nymphs of Triatoma sordida are similar to Rhodnius neglectus and, T. infestans stay very close to $R$. prolixus. genera for thick-walled trichoidea sensilla, and between species of Triatoma for the thin-walled trichoidea (Table III).

Discriminant analysis using variables from adults (Fig. 3) demonstrates a short distance between Rhodnius species (Mahalanobis distance $=54.24)$ compared with Triatoma species (Mahalanobis distance $=409$ ). Comparing genera, the Mahalanobis distance was $359.25(\mathrm{~F}=202.07$, $\mathrm{p}<0.00001)$. In order to check the relative importance of the three antennal segments to separate the four species, the discriminant analysis was repeated using alternatively, variables from the different segments. Fig. 4 shows that variables from the pedicel, were very important in order to separate Triatoma species. Also, the two genera will be easily separated by pedicel, as the Rhodniiini has not these chemo-sensilla. On the other hand, variables of the second flagellar segment were the best to separate Rhodnius species.

As in the fifth nymphal stage, variables from the distal segment of flagellum revealed that $T$. sordida is similar to R. neglectus (Mahalanobis dis-

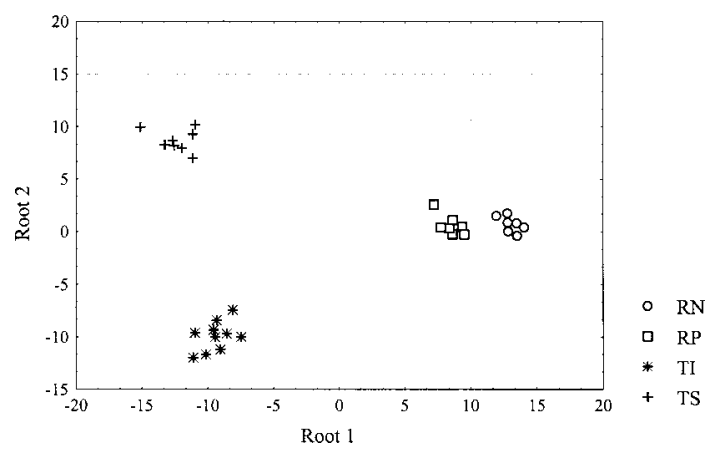

Fig. 3: discriminant analysis performed with 12 variables of the adults antennae. The four species were completely separated. 


\section{TABLE III}

Transitional increment: nymphs to adults, of three types of chemoreceptor sensilla on the antennae of Rhodnius prolixus, $R$. neglectus, Triatoma infestans and $T$. sordida. Each number shows how many times the sensilla number increment from nymphs to adults

\begin{tabular}{lrccc}
\hline & $\begin{array}{c}\text { Triatoma } \\
\text { sordida }\end{array}$ & $\begin{array}{c}T . \\
\text { infestans }\end{array}$ & $\begin{array}{c}\text { Rhodnius } \\
\text { neglectus }\end{array}$ & $\begin{array}{c}R . \\
\text { prolixus }\end{array}$ \\
\hline Thin walled & 17.14 & 11 & 3.98 & 5.14 \\
Thick walled & 6.11 & 3.54 & 2.64 & 2.36 \\
Basiconic & 4.31 & 2.28 & 2.53 & 3.3 \\
\hline
\end{tabular}

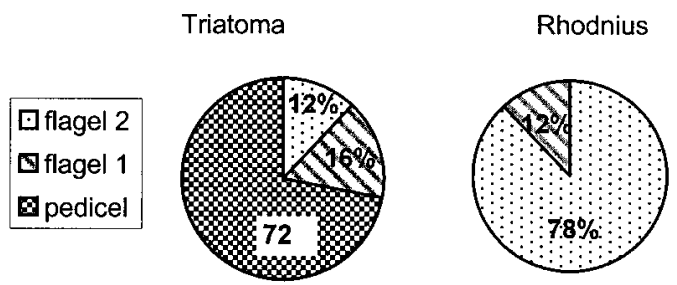

Fig. 4: contribution of each antenna segment to species differentiation in Rhodnius and Triatoma. The figure represent the Mahalanobis distances between species, obtained from discriminant analysis of the sensilla pattern, segment by segment.

tance: 3.04$)$ and, $T$. infestans stay very close to $R$. prolixus (Mahalanobis distance: 1.82). Grouping $T$. infestans plus $R$. prolixus and T. sordida plus $R$. neglectus, the number of thick walled trichoidea differed significatively between groups (mean $[\mathrm{T} . \mathrm{i}+\mathrm{R} . \mathrm{p}]=749, \mathrm{SD}=138$; mean $[\mathrm{T} . \mathrm{s}+\mathrm{R} . \mathrm{n}]=612, \mathrm{SD}=$ $71 ; \mathrm{p}<0.00001)$.

\section{DISCUSSION}

Blood feeding has evolved independently a number of times in the insects (McIver 1987). This has resulted in a great variety of morphological types of sensilla amongst haematophagous insects. In general, the morphology of sensilla reflects taxonomic relationships rather than feeding behaviour. Consequently, blood-feeders have sensilla more similar in structure to their phytophagous or saprophagous close relatives than to distantly related haematophagous species. However, differences in sensitivities and numbers of sensilla, particularly on chemosensilla, become apparent when blood feeders and other insects are compared. Studies from two hemiptera, C. lectularius, an haematophagous Cimicidae (Steinbrecht \& Muller 1976), and Ligus lineolaris a phytophagous Miridae (Chinta et al. 1997) showed morphological types of antennal sensilla very similar to triatomine. But differences are present regarding the distribution and total sensilla number. Within Triatominae, the morphological types of sensilla are similar in all species so far studied, although their relative abundance and distribution vary between species and between nymphs and adults (Catalá \& Schofield 1994, Catalá 1997, Catalá et al. 1998). This paper confirms previous work using not density but total number and distribution of chemoreceptors on the insect antennae.

The use of multivariate linear discriminant analysis (Morrison 1976) had been demonstrated to be potentially useful in Rhodnius species classification and to agree with results from previous biochemical and morphometric studies of these species (Catalá \& Schofield 1994). Our present results, applying discriminant analysis to four species of Rhodnius and Triatoma, confirm that the antennal sensilla pattern is specific and can show very well differences between genera, species, and developmental stages. First, species differentiation is particularly evident in the sensilla pattern of pedicel in Triatoma sp. but not in Rhodnius sp., whose main differences are from the second flagelar segment. The shortest Mahalanobis distances obtained for Rhodnius species compared with Triatoma species, support Schofield's (1988) idea of a monophyletic origin of the Rhodniini and their similitude in habitat and hosts. On the other hand, both species of Triatoma, very well known for their differences in habitats and behaviour (Lent \& Wygodzinsky 1979, Gorla et al. 1993, Schofield 1994) showed important differences in the sensilla pattern.

Zacharuk (1985) points out that in insect antennae, the scape and pedicel bear primarily mechanosensilla and that the greatest number of antennal sensilla, particularly chemosensilla and thermo-hygroreceptors, occurs on the flagellum. In adult insects, the density of sensilla tends to be significantly higher on the more distal than on the proximal segments. This is true for Rhodnius species whose distal flagellar segment bears many more chemoreceptors than the proximal segments. However, Triatoma species show two important differences to Rhodnius species. First, the most populated segment is the proximal segment of the flagellum and second, the pedicel can hold one to three types of chemosensilla (Catalá 1997). These characteristics could be an evidence of a different evolutionary way for Triatoma and Rhodnius species.

Since nymphs and adults occupy the same habitat and feed on the same hosts, the chemo-sensilla transitional difference between nymphs and adults presumably reflects the sexual maturity of adults and/or their dispersive capacity. There are few evidences of sexual dimorphism in the antennal arrays of Triatominae, probably because it had been poorly explored, but Catalá (1997) demonstrated that in $T$. 
sordida, the thin walled trichoidea are more abundant in males than in females. On the other hand, the thick walled trichoidea dominate on the female antenna. These differences were not observed in T. infestans. As all the specimens studied here are males, this may be a good explanation for the highest transitional increment of thin walled trichoidea in T. sordida.

The differences in number and distribution of chemosensilla from nymphs to adults may be in some way associated with the dispersive activities of the adult bugs. This idea is supported by comparison of $T$. sordida with $T$. infestans, the former having a greater capacity for active dispersal and colonisation of new habitats, whereas the latter seems unable to colonise silvatic habitats outside its original silvatic focus in central Bolivia (Schofield 1988, Schofield et al. 1991, 1992). T. sordida has been considered one of the most habitat-generalist species of Triatominae (Schofield 1988) and it tends to show greater population variability (assessed by isoenzymes) compared to other species such as T. infestans. The two species of Rhodnius studied here are believed to have broadly similar capacities for active dispersal and colonisation of new habitats, although in quite different geographic regions. The transitional difference in numbers of chemosensilla is similar for both species.

Moreover, it is noteworthy that in Cimex, whose wingless adults have no greater dispersive capacity than the nymphs, there seems to be no significant transitional difference in the sensilla arrays between nymphs and adults. The general pattern of chemoreceptors in nymphs and adults of Cimex (as illustrated by Steinbrecht \& Müller 1976) is very similar to that of triatomine nymphs studied here.

It has been suggested (Chapman 1982, Zacharuk 1985) that the number of chemoreceptors would be correlated with the length of the antenna. However, this does not seem to be the case with the Triatominae where, for example, $T$. sordida has relatively short antennae (both in absolute terms and in size-corrected terms) but shows a considerably more dense array of chemoreceptors than the other species studied here. The sensorial array represents the primary means of communication between an insect and its environment. As such, key components of the array, such as chemoreceptors, can be expected to reflect the selection pressures that the environment may exert. Discriminant analysis with variables derived from the distal segment of flagellum, showed a similarity between the well domiciliated $R$. prolixus and T. infestans and between the mostly peridomestic and silvatic $R$. neglectus and $T$. sordida. The thick walled sensilla rise significantly in the second flagellar segment of adults and nymphs of $R$. prolixus and $T$. infestans. The function of this sensillum is unknown.

On the other hand, the adults of $R$. prolixus and $T$. infestans showed less thin walled trichoidea than $R$. neglectus and T. sordida. The function of this sensilla may be associated with reproductive activities (Catalá 1997). They respond to a range of fatty acids, particularly pyruvic and lactic, and to amyl acetate (Bernard 1974). The reduction of the sensorial array devoted to reproduction had been predicted by Schofield (1988), as a consequence of domiciliation. He postulated a diminished necessity of seeking a sexual mate in the more stable domestic environment.

\section{ACKNOWLEDGEMENTS}

To Dr CJ Schofield for criticisms and fruitful discussions as well as for the revision of the manuscript. To the Servicio Nacional de Chagas that provided specimens and to TL Victor Tomasi who kindly helped with the antenna preparation.

\section{REFERENCES}

Barret T 1991. Advances in triatomine bug ecology in relation to Chagas disease. Triatominae ecology. $A d v$ Dis Vect Res 8: 144-175.

Bernard J 1974. Étude Electrophysiologique des Récepteurs Impliqués dans l' Orientation vers l'Hôte et dans l'Acte Hematophage chez un Hémiptère Triatoma infestans, Thesis, Université de Rennes, Rennes, 265 pp.

Catalá S 1994. The cave organ of Triatominae bugs. Mem. Inst Oswaldo Cruz 89: 275-277.

Catalá S 1996. Rostral sensilla associated with eight species of Triatominae. J Morphol 228: 195-201.

Catalá S 1997. Antennal sensilla of Triatominae (Hemiptera, Reduviidae): a comparative study over five genera. Int J Insect Morphol Embryio 26: 67-73.

Catalá S, Schofield CJ 1994. The antenal sensilla of Rhodnius. J Morphol 21:193-203.

Catalá S, Tomasi V, Hliba E, Rovasio R 1998. The cave organ of the Triatominae antennae: an olfactory receptor? Biocell 22: 1-7.

Chapman R 1982. Chemoreception: The significance of receptors numbers. Adv Insect Physiol 16: 247-356.

Chinta S, Dickens JC, Baker GT 1997. Morphology and distribution of antennal sensilla of the tarnished plant bug, Lygus lineolaris (Palisot de beauvois) (Hemiptera:Miridae). Int J Insect Morphol Embriol 26: 21-26.

Gorla D, Jurberg J, Catalá S, Schofield C 1993. Systematic of Triatoma sordida, Triatoma guasayana and Triatoma patagonica. Mem Inst Oswaldo Cruz 88: 379-385.

Lazzari CR 1990. Fisiología del Comportamiento de Triatoma infestans (Klug, 1834) (Heteroptera: Reduviidae), Orientación Térmica, Thesis, Universidad Buenos Aires, Buenos Aires, 198 pp.

Lent H, Wygodzinsky P 1979. Revision of the Triatominae (Hemiptera, Reduviidae), and their significance as vectors of Chagas' disease. Bull Amer Mus Nat Hist 163: 1-520. 
Lorenzo Figueiras A, Kenigsten A, Lazzari C 1994. Aggregation in the haematophagous bug Triatoma infestans: Chemical signals and temporal pattern. $J$ Insect Physiol 40: 311-316.

Mayer M 1968. Response of single olfactory cell of Triatoma infestans to human breath. Nature 220: 924-925.

McIver S 1987. Sensilla of haematophagus insects sensitive to vertebrate host-associated stimuli. Insect Sci Applic 8: 627-635.

Morrison D 1976. Multivariate Statistical Methods, Mc Graw-Hill Kogakusha Ltd, Tokio.

Schaller D 1978. Antennal sensory system of Periplaneta americana. Distribution and frequency of morphologic types of sensilla and sex-specific changes during postembryonic development. Cell Tiss Res 191: 121-139.

Schofield C 1988. Biosystematics of the Triatominae, $\mathrm{p}$. 284-312. In MW Service, Biosystematics of Haematophagous Insects, Systematics Association Special 37, Clarendon Press, Oxford.

Schofield C, Lehane M, McEwan P, Catalá S, Gorla D 1991. Dispersive flight by Triatoma sordida. Trans
R Soc Trop Med Hyg 85: 675-678.

Schofield C, Lehane M, McEwan P, Catalá S, Gorla D 1992. Dispersive flight by Triatoma infestans under natural climatic conditions in Argentina. Med Vet Entomol 6: 51-56.

Schofield CJ 1994. Triatominae: Biología y Control, Eurocommunica Publications, $79 \mathrm{pp}$.

Sokal R, Rohlf F 1979. Principios y Métodos Estadísticos en la Investigación Biológica, Blume, Madrid, 831 pp.

Steinbrecht R, Muller B 1976. Fine structure of the antennal receptors on the bed bug, Cimex lectularius L. Tissue Cell 8: 615-636.

Taneja J, Guerin P 1997. Ammonia attracts the haematophagous bug Triatoma infestans: behavioural and neurophysiological data on nymphs. Comp Physiol 181: 21-24.

Wigglesworth V, Gillett J 1934. The function of the antennae in Rhodnius prolixus (Hemiptera) and the mechanism of orientation to the host. J Exp Biol 11: 120-39.

Zacharuk R 1985. Antennae and sensilla, p. 1-70. In LI Gilbert \& GA Kerkut (eds), Comprehensive Insect Physiology, Biochemistry and Pharmacology, Vol. 6, Pergamon Press, Oxford. 